\title{
Paper-Centric Structuring in Learning Processes
}

\author{
Jürgen Steimle, Oliver Brdiczka and Max Mühlhäuser \\ Telecooperation Group, Technische Universität Darmstadt, Germany \\ \{steimle,brdiczka,max\}@tk.informatik.tu-darmstadt.de
}

\begin{abstract}
Structuring constitutes an important means for enhancing individual and collaborative learning processes. This paper introduces two new concepts for augmented semantic structuring of paper-based learning processes. Digital Paper Bookmarks can be used for individually structuring, indexing and tagging learning documents. The Learner Cube provides a new structuring method for collaborative face-to-face learning meetings. Both concepts rely on the Anoto technology, permitting to track and digitalize the paper-based learning process. A first pilot study indicates that Digital Paper Bookmarks and tangible interaction are promising tools for structuring learning processes.
\end{abstract}

\section{Introduction}

Structuring constitutes an important means for enhancing individual and collaborative learning processes. The individual processes of structuring learning material, identifying important passages and assessing own difficulties are important factors for the learning success of students [3]. Structuring is equally important in face-to-face collaborative learning because these involve complex group learning processes and interactions. We therefore seek for efficient methods enabling students to structure both learning material and group learning processes with semantic types.

Empirical work [4] indicates that in many settings, paper is the preferred learning medium. Interacting with printed documents with pen and paper has intrinsic advantages over interacting with a computer, such as two-handed interaction, tactile feedback and ease of annotation. We depart from these findings and augment paper-based tangible interaction by computer support for sharing and aggregating structuring results.

In this paper, we introduce two new paper-based interaction concepts for augmented structuring and corresponding digital support in individual and collaborative learning processes: Digital Paper Bookmarks and the Learner Cube. Digital Paper Bookmarks combine the advantages of paper-based bookmarking with electronic support for individual learning processes. The Learner Cube is a paper-based cube for structuring and enhancing collaborative learning processes with electronic support. Prototype systems have been built for both concepts. A first pilot study shows that Digital Paper Bookmarks are promising tools for the paperbased structuring of individual learning processes.

\section{Augmented Structuring in Learning Processes}

For the paper/digital synchronization, our system relies on the Anoto technology [1]. This uses a specific dot pattern printed on paper. The pattern enables an electronic pen to identify both the page and its position on this page. In contrast to other solutions for capturing handwriting on paper, no specific sensing device other than the electronic pen is needed. This provides for a natural writing and switching between paper sheets like with traditional pen and paper.

\subsection{Structuring in Individual Learning Pro- cesses: Digital Paper Bookmarks}

Paper bookmarks are a very efficient means for structuring documents, marking specific passages on paper and quickly accessing them later on [5]. In order to offer electronic support, we developed Digital Paper Bookmarks which combine the advantages of paperbased bookmarking with electronic support.

Digital Paper Bookmarks are Post-it like adhesive stickers of different colors which can be attached to physical pages of printed documents at arbitrary positions (Figure 1 left). Digital Paper Bookmarks are covered with Anoto pattern and can therefore be labeled with a title using an electronic pen. They are synchronized with the electronic system and serve as electronic bookmarks for these pages. Users can easily create Digital Paper Bookmarks via three steps (Attach Associate - Label). First, a bookmark can be attached to arbitrary positions at any page of the printed docu- 
ment. Then, the paper bookmark is associated with the page by drawing a short line connecting the bookmark with the page. Finally, since the Anoto dot pattern is printed on the bookmark, users can use the electronic pen to write a tag or title on it. Once created, a Digital Paper Bookmark can be modified by sticking it to another position and performing the association gesture again. It can be deleted by removing and writing a cross out deleting gesture on it.

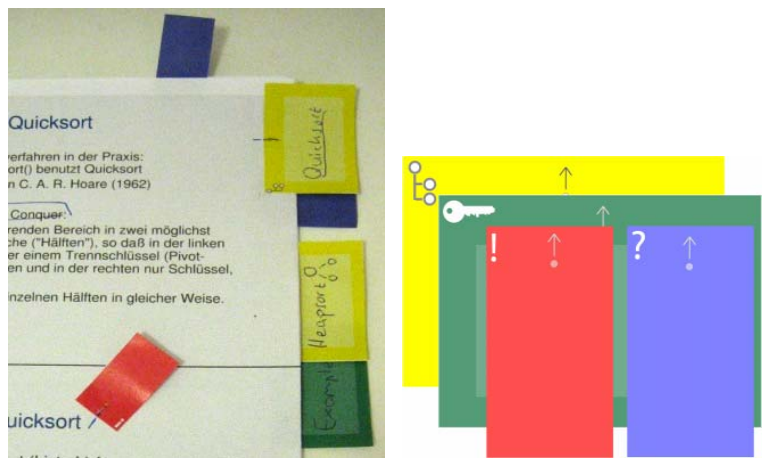

Figure 1. Digital Paper Bookmarks in a printed document (left) and semantic types (right)

Semantic Types: In order to structure learningrelated bookmarking processes, Digital Paper Bookmarks can express several semantic types (Figure 1 right). Section bookmarks (yellow) can be used to mark the beginning of a new section. The title of this section can be written on the bookmark. Key bookmarks (green) can be used to mark central passages of a section, where subject matters are defined or explained. Important bookmarks (red) are used to mark passages which seem particularly relevant to the learner. Unclear bookmarks (blue) are used to point out passages which the learners have difficulties understanding and which therefore require further work. Several bookmarks can be attached to the same page, allowing for example marking a definition with the name of the subject matter and to underscore its particular relevance with an additional red important bookmark.

The proposed solution for creating bookmarks is entirely mobile and does not require any changes from traditional paper-based work.

\subsection{Structuring in Collaborative Learning Pro- cesses: The Learner Cube}

Small learning group meetings are complementary to the individual learning process in many aspects. Collaborative face-to-face learning processes additionally provide achievement gains, promote critical thinking and reflection, and enhance problem solving capabilities [6]. In order to structure and augment the collaborative learning process, we propose a new concept: the Learner Cube. It is a wooden cube of $9.5 \times 9.5 \times 9.5 \mathrm{~cm}$ that is entirely covered with paper containing the Anoto pattern (Figure 2 left). On each side of the cube, the paper covering has a different color, representing different phases in the collaborative learning process.

Collaborative face-to-face learning in small groups augments the learning process by an additional dimension: interaction. While paper documents and bookmarks are rather used for individual learning processes, structuring a collaborative learning process requires a shared object. This object can be manipulated by all participants and therefore helps in jointly structuring the collaborative learning process. We opt for a tangible 3D cube because it provides for a very intuitive, shared manipulation and gives equal weight to all viewpoints of the participants located around it. Furthermore, the top side can be interpreted as the actual state of the cube (and thus of the learning process). As other tangible objects offer similar affordances, these could also be appropriate means for structuring collaborative processes. We leave this for future work.

Covering up to six important phases in group learning processes, the sides of the cube stimulate learners to perform these phases. Each time the group proceeds to a subsequent phase, the cube can be rotated to have the corresponding side lying on top. This manipulation materializes the meta-processes in group learning and supports negotiation and explicit and joint decisions.

In addition to the semantic phases represented by the sides of the cube, digital paper Post-its can be attached to the sides of the cube (see Figure 2 left). This supports collaboratively collecting semantic items that are important and specific to this actual phase, e.g. relations to a specific learning content. Like Digital Paper Bookmarks, the Post-its are covered with Anoto pattern and can be labeled with a title.

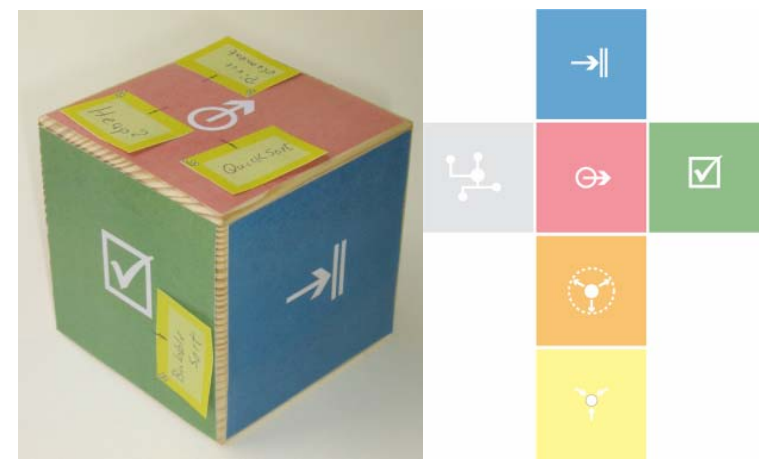

Figure 2. Learner Cube (left) and semantic types (right)

Semantic Types: Based on existing models for structuring collaborative learning [2], we distinguish six semantic phases of the learning process (Figure 2):

1. Startup (red): participants gather together and identify the topics of the meeting; Post-its represent topics

2. Explaining (yellow): for the basic understanding of a topic, definitions/explanations are discussed; 
Post-its represent important properties of the topic

3. Elaboration (orange): recall cues are collected (e.g. additional details, generated examples and images); Post-its represent recall cues for the topic

4. Abstraction (gray): a topic is put in relation to existing knowledge and the entire learning content; Post-its represent links and levels of abstraction

5. Successfully accomplished (green): contains the topics that have been successfully discussed and accomplished; Post-its represent topics

6. Ending/To Do (blue): end phase of the meeting; agreement on tasks to perform individually after the meeting; Post-its represent "to do"-items

Depending on the group processes to support, the cube can be easily modified to support other semantic types. For later electronic retrieval, RFID tags inside the cube and the Anoto technology enable the tracking of each learning phase as well as of semantic associations on Post-its.

\section{Digital Support}

A computer system can generate visualizations of the individual and collaborative learning processes. Paper bookmarks can be integrated to and displayed within the corresponding digital document. This creates a personalized index of contents of the document. A collaborative visualization, which integrates own and shared bookmarks of other learners, enables learners to critically examine their own understanding by comparing their own structure with those of other learners. Cognitive conflicts may arise and can lead to a modification of one's own bookmarks. Figure 3 depicts our collaborative visualization of Digital Paper Bookmarks, where bookmarks are displayed beneath an abstract representation of the document pages. We currently work on the design of a visualization of group processes which are structured with the Learner Cube.

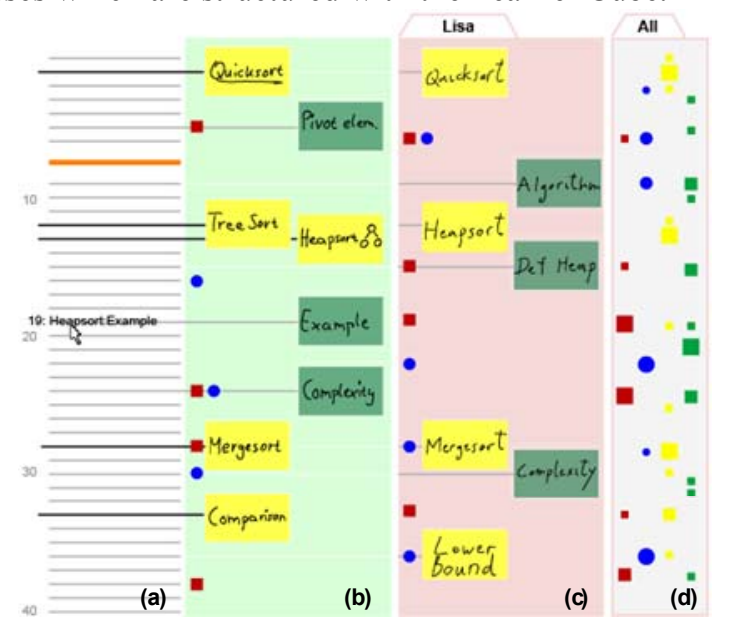

Figure 3. Visualization of own bookmarks (b), shared bookmarks (c) and an aggregated view (d).

\section{First Evaluation \& Conclusion}

As a first technique of paper-based structuring, we implemented Digital Paper Bookmarks and conducted a pilot study. We asked nine randomly selected students attending an introductory computer science lecture to structure printed slides of one lecture with Digital Paper Bookmarks. Our observations and semistructured interviews with the participants indicate that Digital Paper Bookmarks are very intuitive to create and modify $(\mathrm{M}=4.7$ on a 5 -point Likert scale, $\mathrm{SD}=.5)$. They judged Digital Paper Bookmarks as very helpful for finding specific pages $(\mathrm{M}=4.4, \mathrm{SD}=.5)$ as well as for orientation in the paper stack $(\mathrm{M}=4.7, \mathrm{SD}=.5)$. Several participants particularly valued the possibility to fade away from the given structure and instead to create an own structure of the document. Based on this encouraging feedback, we will conduct subsequent studies over a longer period of time assessing both the asynchronous process of sharing bookmarks and the use of the Learner Cube.

We believe that Digital Paper Bookmarks and the Learner Cube constitute new concepts for the nonobtrusive, tangible and intuitive structuring in individual and collaborative learning processes. Our first study confirms that Digital Paper Bookmarks seem to be very promising tools for structuring documents in learning settings. Although our current work focuses on learning, our concepts are applicable to more general settings like document indexing in knowledge work and group processes in meetings. In our ongoing work, we will explore additional means for tangible structuring, computer visualization and computer mediation to fully leverage the power of paper-centric structuring in learning individual and collaborative learning.

\section{References}

[1] Anoto. http://www.anoto.com.

[2] Alison King. Scripting Collaborative Learning Processes: A Cognitive Perspective, chapter 2, pages 13-37, CSCL, Springer, 2007.

[3] M. Kliegel, M. Altgassen, M. Martin, and A. Kruse. Lernen im Alter: Die Bedeutung der selbstständigen Strukturierung des Lernmaterials. Zeitschrift für Gerontologie und Geriatrie, 36:421-428, 2003.

[4] A. Sellen and R. Harper. The Myth of the Paperless Office. MIT Press, Cambridge, 2003.

[5] M. Thakor, W. Borsuk, and M. Kalamas. Hotlists and web browsing behavior - an empirical investigation. Business Research 57:776-786, 2004.

[6] N.M. Webb and A.S. Palincsar. Group processes in the classroom, pages 841-873. Handb. Educational Psychology. Macmillan, 1996. 